\title{
MicroRNA-194 promotes the growth, migration, and invasion of ovarian carcinoma cells by targeting protein tyrosine phosphatase nonreceptor type 12
}

This article was published in the following Dove Press journal:

OncoTargets and Therapy

15 July 2016

Number of times this article has been viewed

\section{Tian Liang \\ Liru Li \\ Yan Cheng \\ Chengcheng Ren \\ Guangmei Zhang}

Department of Gynecology and Obstetrics, The first Affiliated Hospital of Harbin Medical University, Nangang District, Harbin, Hei Longjiang, People's Republic of China
Correspondence: Guangmei Zhang Department of Gynecology and Obstetrics, The First Affiliated Hospital of Harbin Medical University, 23 Youzheng Street, Nangang District, Harbin, Hei Longjiang, People's Republic of China Email guangmeizhang194@I26.com

\begin{abstract}
Ovarian carcinoma is the most lethal gynecologic malignancy among women. Ovarian cancer metastasis is the main reason for poor prognosis. MicroRNAs (miRNAs) have been shown to play an important role in tumorigenesis and metastasis in various cancers by affecting the expression of their targets. In this study, we explored the role of miR-194 in ovarian cancer. Real-time polymerase chain reaction assays showed that miR-194 was significantly upregulated in ovarian cancer tissues. Overexpression of miR-194 in ovarian cancer cells promotes cell proliferation, migration, and invasion; in contrast, inhibition of the expression of miR-194 has the opposite effects. Meanwhile, bioinformatics tools were used to identify protein tyrosine phosphatase nonreceptor type 12 (PTPN12) as a potential target of miR-194. The luciferase assay showed that miR-194 directly binds to the 3'-untranslated region of PTPN12. Western blot analysis and quantitative real-time polymerase chain reaction assay revealed that PTPN12 expression was negatively associated with miR-194 expression in both ovarian cancer tissues and cells. Thus, we conclude that miR-194 targets PTPN12 and functions as an oncogene in ovarian cancer cells. This novel pathway may provide a new insight to explain ovarian cancer development and metastasis.
\end{abstract}

Keywords: miR-194, ovarian cancer, PTPN12, metastasis

\section{Introduction}

Ovarian cancer is the most common cause of gynecologic malignancy-related mortality in women. ${ }^{1}$ According to the report of the National Cancer Institute, 21,290 new cases will be diagnosed and $>14,180$ patients will die in the US in 2015 . In addition, $\sim 67 \%$ of patients with ovarian cancer are diagnosed in late stage because of lack of apparent symptoms in early stage. ${ }^{2}$ This means that extensive abdominal metastasis has been formed when the disease is diagnosed, which is the main reason for the high mortality of ovarian cancer. ${ }^{3}$ Thus, inhibition of metastasis of ovarian cancer cells after diagnosis is helpful for changing the poor prognosis. However, the mechanisms of ovarian cancer metastasis have not been fully elucidated, which have thus become the focus of recent research.

MicroRNAs (miRNAs) are a class of small (19-25 nt in length), endogenous, noncoding RNAs that play regulatory functions by binding imperfectly complementary to the $3^{\prime}$-untranslated region (3'UTR) of their target messenger RNAs (mRNAs), controlling the translation of mRNA to protein. ${ }^{4,5}$ miRNAs are involved in regulating cellular processes, such as proliferation, differentiation, and apoptosis. ${ }^{5}$ Moreover, increasing evidence indicated that miRNAs play a significant role in tumor occurrence 
and development $t^{6,7}$ and function as tumor suppressor in oncology. ${ }^{8-10}$

Recently, numerous studies have reported that aberrant level of miR-194 is involved in several cancers, such as endometrial cancer, ${ }^{11}$ colon cancer, ${ }^{12-14}$ and gastric cancer. ${ }^{15}$ The function of miR-194 in different cancers is often associated with its varying targets, and various targets of miR-194 have been found, including BMI-1, ${ }^{11}$ thrombospondin-1, ${ }^{12}$ and FoxM1. ${ }^{15}$ Especially, in pancreatic ductal adenocarcinoma cells, miR-194 overexpression increased tumor growth and invasion through the suppression of DACH1. ${ }^{16}$ Meanwhile, miR-194 could attenuate paclitaxel resistance of ovarian cancer by inhibiting BMI-1 expression. ${ }^{17}$ Studies assessing whether abnormal expression of miR-194 is related to ovarian cancer and on its mechanisms of function are still limited.

Protein tyrosine phosphatases (PTPs), encoded by phosphatase genes, play a critical role in the regulation of cell signaling, cell-cell junctions, and cell cycle progression. ${ }^{18-20}$ Protein tyrosine phosphatase nonreceptor type 12 (PTPN12), located in 7q11.23, is a member of the PTP family. ${ }^{21}$ Recent studies indicated that PTPN12 may be a novel tumor suppressor in breast cancer, esophageal squamous cell carcinoma, and ovarian cancer. ${ }^{22-24}$ Recently, a link between miR-194 and PTPN12 was identified by biological information analysis. ${ }^{25}$ In addition, miR-194 could repress PTPN12, and miR-194 inhibitor could significantly release the repression of PTPN12 through $3^{\prime}$ UTRs. ${ }^{26}$ Therefore, PTPN12 was chosen as a target of miR-194 for our experiments, and a close relation between miR-194 and PTPN12 was suspected in the growth, migration, and invasion of ovarian carcinoma.

Our study demonstrates that the expression of miR-194 was upregulated in ovarian cancer tissue. In addition, miR194 increased the proliferation, migration, and invasion in ovarian cancer cells. More importantly, miR-194 directly targets the 3'UTR of PTPN12 mRNA in ovarian cancer cells. Thus, our findings may provide a novel pathway and molecules as biological makers and targets on ovarian cancer metastasis.

\section{Materials and methods}

\section{Cell lines and cell culture}

Human ovarian cancer cell lines, SKOV3 and OVCAR3, were maintained in Roswell Park Memorial Institute (RPMI)1640 medium supplemented with $10 \%$ fetal bovine serum (FBS; PAA, Austria) at $37^{\circ} \mathrm{C}$ in $5 \% \mathrm{CO}_{2}$ and routinely passaged at 2- to 3-day intervals.

\section{Processing of patient samples with ovarian cancer}

A total of 15 pairs of ovarian cancer tissues and normal ovary tissues were collected from the same patient under surgery at the First Affiliated Hospital of Harbin Medical University after written informed consent was obtained from each patient, and the study was approved by the Ethics Committee of the First Affiliate Hospital of Harbin Medical University. Samples were immediately frozen in liquid nitrogen for subsequent experiments. The cancer samples were diagnosed with ovarian carcinoma based on histopathological evaluation. All cancers were staged according to the International Federation of Gynecology and Obstetrics criteria, and all patients were without chemotherapy before operation. The information and the cancer stage of each patient are listed in Table 1.

\section{RNA isolation, reverse transcription PCR, and real-time PCR}

Total RNA was extracted from ovarian cancer cell lines and ovarian tissues using TRizol reagent (Thermo Fisher Scientific, Waltham, MA, USA) according to the manufacturer's protocol. The integrity of isolated RNA was evaluated by the Agilent Bioanalyzer 2100 with RNA 6000 Nano kit (Agilent Technologies, Santa Clara, CA, USA). Single-stranded complementary DNA was synthesized from $1 \mu \mathrm{g}$ RNA in a $20 \mu \mathrm{L}$ reaction volume using the HighCapacity cDNA Reverse Transcription Kits (Thermo Fisher

Table I Clinicopathological information of 15 ovarian cancer samples

\begin{tabular}{lllll}
\hline Number & $\begin{array}{l}\text { Age of } \\
\text { patients } \\
\text { (years) }\end{array}$ & $\begin{array}{l}\text { FIGO } \\
\text { stage }\end{array}$ & Histotype & Grade \\
\hline I & 47 & II & Endometrioid carcinoma & Moderate \\
2 & 49 & I & Serous cystadenocarcinoma & Well \\
3 & 52 & I & Endometrioid carcinoma & Moderate \\
4 & 55 & III & Serous cystadenocarcinoma & Moderate \\
5 & 57 & II & Mucinous cystadenocarcinoma & Moderate \\
6 & 58 & II & Serous cystadenocarcinoma & Moderate \\
7 & 59 & IV & Endometrioid carcinoma & Poor \\
8 & 61 & II & Serous cystadenocarcinoma & Well \\
9 & 62 & I & Serous cystadenocarcinoma & Moderate \\
I0 & 42 & I & Serous cystadenocarcinoma & Well \\
II & 55 & II & Serous cystadenocarcinoma & Moderate \\
I2 & 48 & II & Endometrioid carcinoma & Moderate \\
I3 & 5 I & I & Serous cystadenocarcinoma & Well \\
14 & 62 & III & Mucinous cystadenocarcinoma & Moderate \\
I5 & 57 & I & Serous cystadenocarcinoma & Well \\
\hline
\end{tabular}

Abbreviation: FIGO, Federation of Gynecology and Obstetrics. 
Scientific), and the reaction was performed according to the manufacturer's protocol. Real-time quantification of miRNA and mRNA was performed using a SYBR Green PCR kit (Thermo Fisher Scientific), and the cycle threshold $(\mathrm{Ct})$ of each gene was recorded. The relative expression of miR-194 and PTPN12 mRNA was normalized to U6 and GAPDH, respectively, and calculated using the $2^{-\Delta \Delta \mathrm{Ct}}$ method $(\Delta \mathrm{Ct}=$ $\left.\mathrm{Ct}^{\text {target gene }}-\mathrm{Ct}^{\text {internal control }}\right){ }^{27}$ The real-time polymerase chain reaction (PCR) conditions were as follows: $95^{\circ} \mathrm{C} 10$ minutes; 40 cycles of $95^{\circ} \mathrm{C} 15$ seconds, $67^{\circ} \mathrm{C} 30$ seconds, $72^{\circ} \mathrm{C}$ 30 seconds, and $72^{\circ} \mathrm{C} 5$ minutes. Primer sequences used in our study are listed in Table 2 .

\section{miRNA transfection}

miR-194 mimics and miR-194 inhibitor were synthesized by GenePharma (Shanghai, People's Republic of China). A total of $5 \times 10^{5}$ ovarian cancer cells were seeded in each well of sixwell plates and cultured in serum-free RPMI-1640 medium for 1 day before transfection. The cells were transfected with $50 \mathrm{nM}$ miR-194 mimics or miR-194 inhibitor by using Lipofectamine ${ }^{\circledR}$ 2000 reagent (Thermo Fisher Scientific), following the manufacturer's protocol. After transfection, real-time PCR assay was used to verify the transfection ratio. Cells transfect with miR-194 mimics or miR-194 inhibitor named as miR-194 mimics group or miR-194 inhibitor group, respectively, and nontransfection cells used as control group are named blank group.

\section{Cell growth curve}

Cell proliferation was assessed by a tetrazolium salt (WST-8)based colorimetric assay provided by the Cell Counting Kit 8 (CCK-8; Biosharp, People's Republic of China). Treated and untreated cells were seeded in 96-well plates $(2,000$ cells

Table 2 qRT-PCR primers and miR-194 mimics and inhibitor sequences used in our study

\begin{tabular}{ll}
\hline Primer & Sequence $\left(5^{\prime} \mathbf{3}^{\prime}\right.$ ) \\
\hline miR-194 RT & GTCGTATCCAGTGCAGGGTCCGAGGT \\
U6 RT & CGCGCACTGGATACGACTCCACA \\
miR-194 forward & GCGGCGGTGTAACAGCAACTCC \\
miR-194 reverse & ATCCAGTGCAGGGTCCGAGG \\
U6 forward & GCTTCGGCAGCACATATACTA \\
U6 reverse & CGCTTCACGAATTTGCGTGTC \\
PTPNI2 rorward & GCAGGAACAACACATTCAGG \\
PTPNI2 reverse & TCCATTCCGATCTTACAGGTG \\
miR-194 mimics & UGUAACAGCAACUCCAUGUGGA \\
miR-194 inhibitor & UCCACAUGGAGUUGCUGUUACA \\
\hline
\end{tabular}

Abbreviations: $\mathrm{QRT}-\mathrm{PCR}$, quantitative real-time polymerase chain reaction; miR 194, microRNA-194; PTPNI2, protein tyrosine phosphatase nonreceptor type 12. per well) after 48 hours posttransfection with $100 \mu \mathrm{L}$ full culture medium. Cell proliferation was assessed once daily for 5 consecutive days after seeding. At each time, $10 \mu \mathrm{L}$ of CCK-8 solution was added to each well, and the plates were incubated for 4 hours in $37^{\circ} \mathrm{C}$. Absorbance was read at a wavelength of $450 \mathrm{~nm}$ by a microplate reader (Infinite ${ }^{\circledR}$ M1000 PRO; TECAN, Switzerland). Growth curves were constructed by plotting absorbance values versus time (days). The experiments were repeated three times.

\section{Wound healing assay}

Migration of treated and untreated ovarian cancer cells was detected by an in vitro wound healing assay. The cells were cultured with serum-free RPMI-1640 media till they grow to $90 \%-95 \%$ confluence in six-well plates, and then scratch wounds were produced with $10 \mu \mathrm{L}$ pipette tips in each well. The scratch wounds were observed by phase-contrast microscope (Nikon, TS100; Nikon Corporation, Tokyo, Japan) after 0 and 24 hours, respectively. Photographs were taken to access the level of migration in each group of the transfected cells.

\section{Transwell invasion assay}

Cell invasion assay was carried out using BIOCOAT Matrigel Transwell chamber (BD, Franklin Lakes, NJ, USA) with the pore size of $8.0 \mu \mathrm{m}$. The inserts were placed into 24-well plates, containing $700 \mu \mathrm{L}$ of RPMI-1640 medium for 30 minutes in a humidified $37^{\circ} \mathrm{C}$ incubator under $5 \% \mathrm{CO}_{2}$ before seeding cells. After 48 hours transfection, $5 \times 10^{4}$ cells in each group resuspended in RPMI-1640 medium containing $5 \%$ FBS were placed in each chamber. The lower compartment was loaded with full media containing $15 \%$ FBS as the nutritional attractant. After being incubated at $37^{\circ} \mathrm{C}$ for 48 hours, noninvaded cells were scraped off with a cotton swab. The translocated cells on the bottom of upper chamber membrane were fixed with 5\% formaldehyde and stained with 1\% Giemsa stain. Five fields of fixed cells were randomly chosen and counted under a light microscope.

\section{Western blot analysis}

The cells were harvested after 72 hours of transfection, washed twice with phosphate-buffered saline, and lysed in radio immunoprecipitation assay (RIPA) lysis with $0.01 \%$ protease and phosphatase inhibitor, respectively, and incubated on ice for 30 minutes. Cell lysis was centrifuged at $14,000 \mathrm{rpm}$ for 15 minutes, and the supernatant $(50 \mu \mathrm{g})$ 
of total protein was run on $10 \%$ sodium dodecyl sulfatepolyacrylamide gel electrophoresis and transferred electrophoretically to a polyvinylidene fluoride membrane (EMD Millipore, Billerica, MA, USA). After blocking in Western Blocking Reagent (10\%; Hoffman-La Roche Ltd, Basel, Switzerland), the membrane was incubated overnight at $4^{\circ} \mathrm{C}$ with polyclonal rabbit anti-human PTPN12 (1:500; Abcam, ab90641, USA) and mouse anti-human GAPDH (1:1,000, Hoffman-La Roche Ltd), respectively, and then the blot was incubated with DyLight ${ }^{\circledR}$-conjugated secondary antibody (Rockland, ME, USA) at room temperature for 1 hour, then visualized and analyzed using the Odyssey IR imaging system (LI-COR Biosciences, Lincoln, NE, USA).

\section{Luciferase reporter assay}

Luciferase reporter assays were performed using the Luciferase Reporter Assay System (Promega Corporation, Fitchburg, WI, USA), according to the manufacturer's instructions. A fragment at 156 bp containing seven miR-194 binding sites of PTPN12 3'UTR (wild type [WT]) or deleting the sequence of binding site (mutant [MUT]) was cloned into the pGL3 vector (Promega Corporation) through restriction enzyme cutting site of NcoI (CCATGG). For the reporter assay, SKOV3 cells were cultured in 24-well plates, transfected with either PGL3-PTPN12-WT or PGL3-PTPN12MUT vector and miR-194 mimics or negative control (NC). After 48 hours, the luciferase activity was measured and normalized to the renilla luciferase activity.

\section{Statistical analysis}

Data were presented as mean \pm standard deviation (SD), in at least three independent experiments. All statistical analyses were performed using SPSS 17.0 statistical software (SPSS Inc., Chicago, IL, USA). The difference between the

A

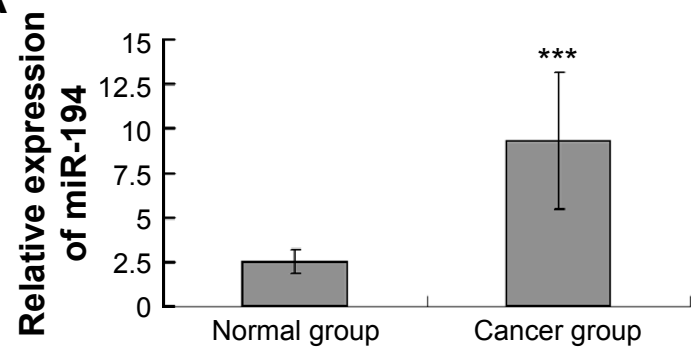

variables was assessed by two-tailed Student's $t$-test and one-way analysis of variance. A $P$-value $<0.05$ is considered significant.

\section{Results}

\section{miR-194 is upregulated and negatively associated with PTPNI 2 in ovarian cancer tissues}

The expression of miR-194 in 15 ovarian cancer tissues and normal ovarian epithelium tissues was quantitated by realtime PCR. We found that compared to normal ovarian epithelium tissues, ovarian cancer tissues expressed higher miR-194 $(P<0.001$; Figure 1A), suggesting that miR-194 has increased expression in ovarian cancer tissues and may be associated with ovarian cancer carcinogenesis. Then we predicted the putative targets of miR-194 by three web-based algorithms, miRWalk, TargetScan, and PicTar (http://www.umm.uniheidelberg.de/apps/zmf/mirwalk2/index.html, http://www. targetscan.org, and http://pictar.mdc-berlin.de/). PTPN12, a tumor suppressor protein, is chosen for our further study. Real-time PCR was performed to analyze the expression of PTPN12 mRNA in ovarian cancer and normal ovarian tissues. Results showed that PTPN12 mRNA was downregulated in ovarian cancer tissues $(P<0.001$; Figure 1B). Therefore, we conclude that miR-194 is upregulated in ovarian cancer tissues and negatively associated with PTPN12.

\section{miR-I94 enhances cell growth in vitro}

First, SKOV-3 and OVCAR-3 cells were transfected with miR-194 mimics or miR-194 inhibitor; real-time PCRs were performed to verify the transfection ratio (Figure $2 \mathrm{~A}$ and $\mathrm{B}$ ). Then, CCK-8 assays were performed to investigate the effects of miR-194 on cellular proliferation in ovarian cancer cells. The results indicated that overexpression of miR-194 can

B

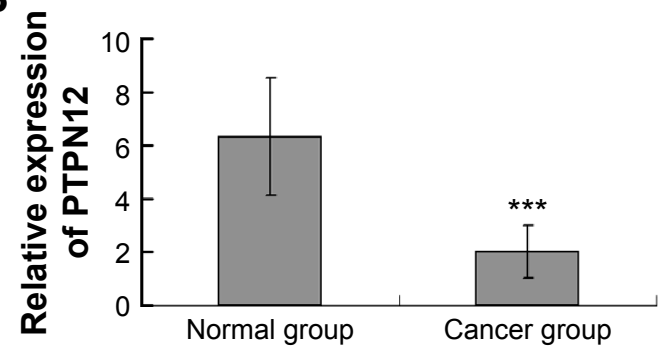

Figure I miR-194 shows high expression in ovarian cancer tissues and is negatively associated with PTPNI2.

Notes: (A) qRT-PCR level of miR-194 expression in ovarian cancer tissues $(n=15)$ and normal ovary tissues $(n=15)$. Significantly higher expression of miR-194 was detected in ovarian cancer tissues than in normal ovary tissues $(* * * P<0.00 \mathrm{I})$ and $(\mathbf{B})$ relative expression of PTPNI 2 mRNA was lower in cancer group $(* * * P<0.00 \mathrm{I})$.

Abbreviations: qRT-PCR, quantitative real-time polymerase chain reaction; miR-194, microRNA-194; PTPNI2, protein tyrosine phosphatase nonreceptor type 12; mRNA, messenger RNA. 

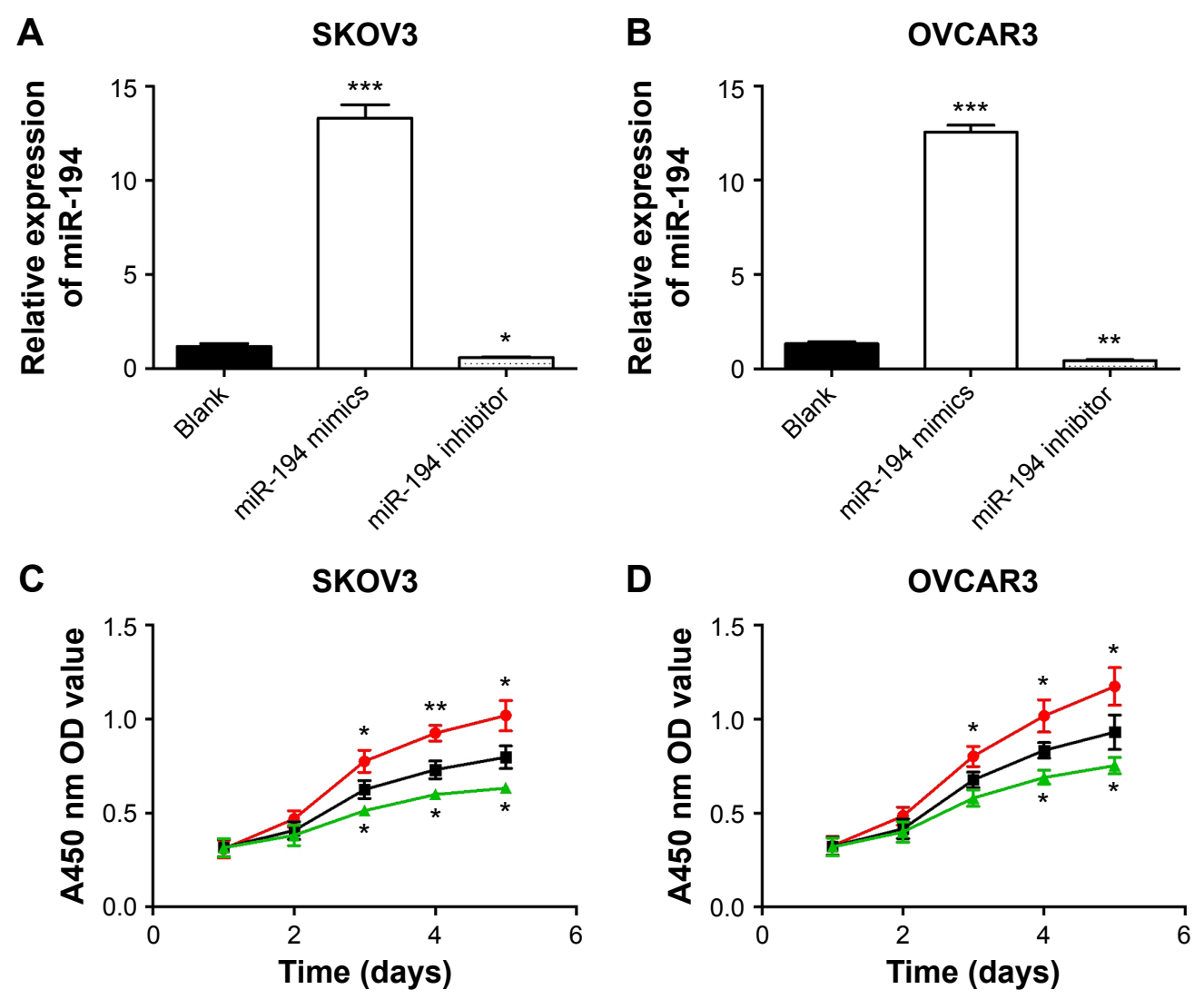

- Blank $\rightarrow$ miR-194 mimics $₫$ miR-194 inhibitor

Figure 2 miR-194 significantly enhanced the proliferation of ovarian cancer cells (SKOV3 and OVCAR3).

Notes: After transfection with oligonucleotides, the relative expression of miR-194 in miR-194 mimics group, miR-194 inhibitor group, and blank group was as shown in $(\mathbf{A}$ and $\mathbf{B})$. Cell growth curve of both cell lines indicated that upregulated miR-I94 enhanced cell proliferation, and cell growth was decreased by miR-I94 inhibitor (C and $\mathbf{D})$. $* P<0.05 ; * * P<0.01 ; * * * P<0.001$.

Abbreviations: miR-194, microRNA-194; OD, optical density.

significantly enhance the proliferation of ovarian cancer cells in both cell lines (Figure 2C and D).

\section{miR-194 increased cell migration in ovarian cancer cell lines}

We observed the effects of miR-194 on cell migration of ovarian cancer cells by wound healing assay. The wound healing scratches were observed by phase-contrast microscope at 0 and 24 hours, which showed that exogenetic expression of miR-194 caused a significantly increased capacity of cell migration in both cell lines (Figure 3).

\section{miR-194 enhanced invasion in ovarian cancer cells}

Furthermore, cell invasion was verified by transwell assay. The results of transwell invasion assay illustrated that miR-194-overexpressed SKOV-3 and OVCAR-3 cells had the higher potential of invasion when compared with the control group and miR-194-downregulated cell groups (Figure 4).

\section{PTPNI 2 is a direct target of miR-194 in ovarian cancer cells}

Publicly available algorithms (PicTar, TargetScan, and miRWalk) were used to predict the target of miR-194; we found the 3'UTR of PTPN12 containing a target sequence for miR194 at nt 228-235 (Figure 5A). To verify whether miR-194 target the 3'UTR of PTPN12 mRNA in ovarian cancer cells, we cloned the target sequence of PTPN12 3'UTR (WT) or the mutant sequence (MUT) into a luciferase reporter vector and cotransfected these reporter vectors into SKOV-3 cells with miR-194 mimics or miRNA controls. We found that the overexpression of miR-194 reduced the luciferase activity in cells transfected with PGL3-PTPN12-WT but no significant change in cells with PGL3-PTPN12-MUT (Figure 5B). Similarly, through Western blot assay, we found 

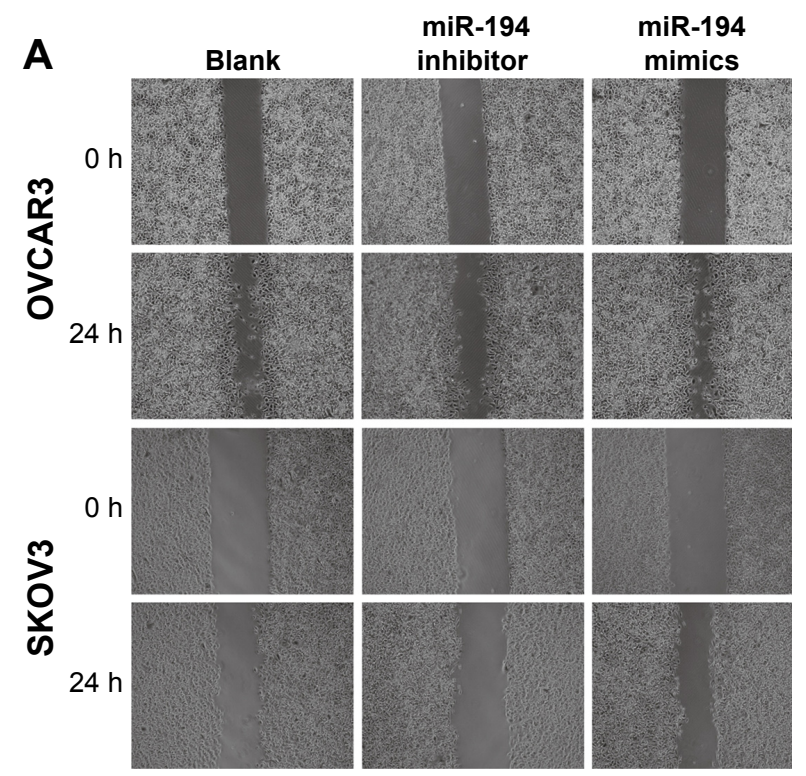

\section{B}

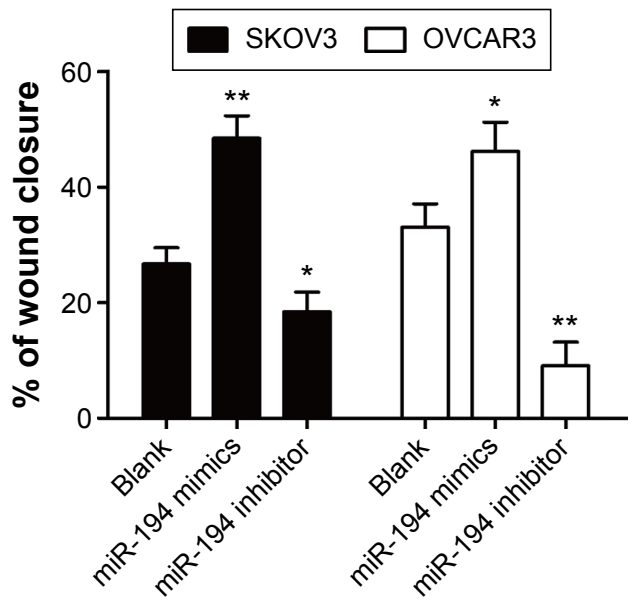

Figure 3 miR-194 increased cell migration in ovarian cancer cells.

Notes: (A and B) Representative images from the results of wound healing assay in SKOV3 and OVCAR3 cells. $* P<0.05$ and $* * P<0.01$.

Abbreviations: miR-194, microRNA-194; h, hour.
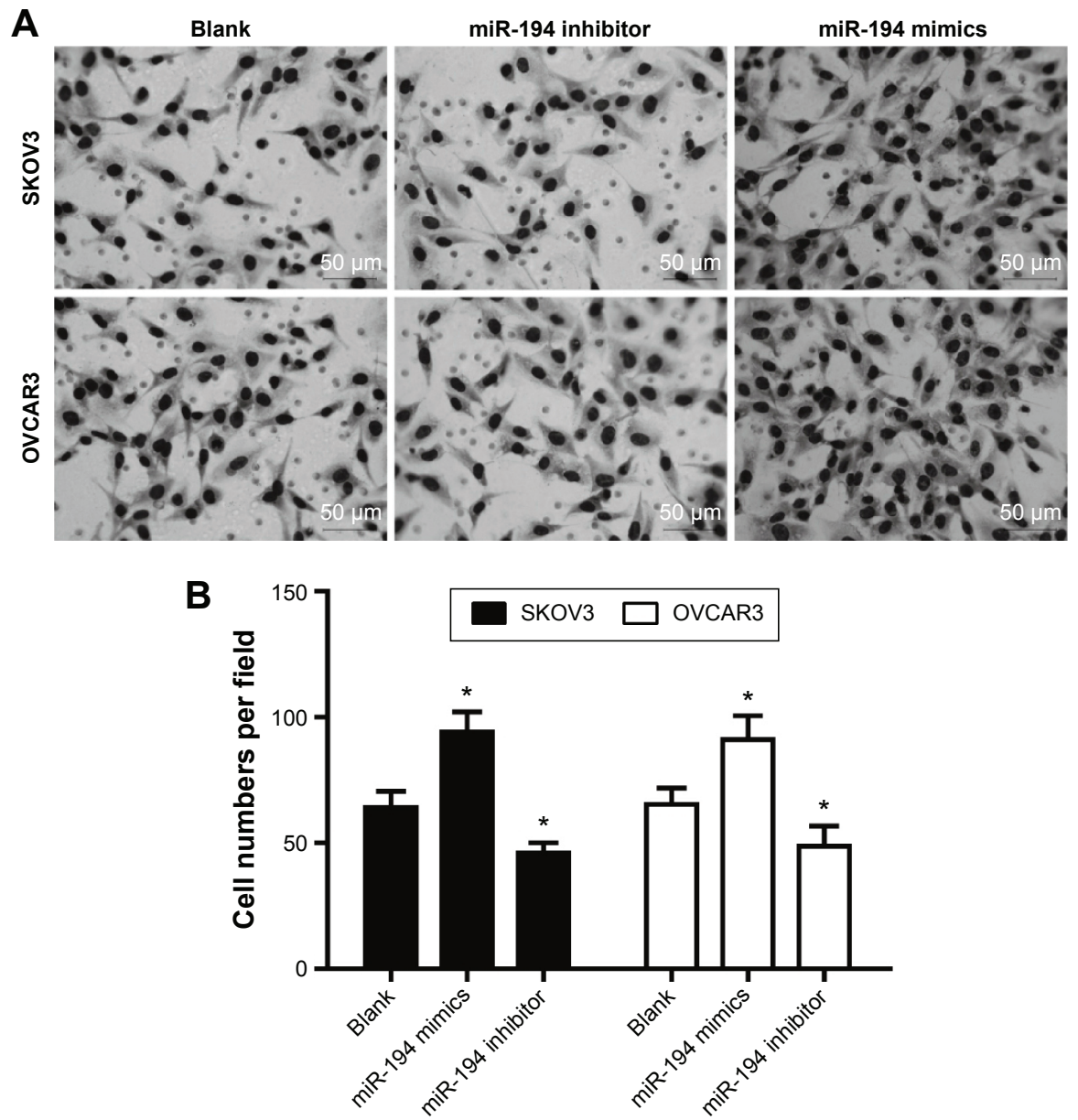

Figure 4 miR-194 enhanced cell invasion in SKOV3 cells and OVCAR3 cells.

Notes: (A) After SKOV3 and OVCAR3 cells were transfected with miR-194 mimics or miR-194 inhibitor for 48 hours, the transwell chambers were stained with Giemsa stain and counted at the same magnification (scale bar equals $50 \mu \mathrm{m}$ ). (B) The number of invasion cells of ovarian cancer cells with miR-194 were overexpressed much more than the blank group; meanwhile, miR-194 inhibitor group shows a decrease in invasion capacity $(* P<0.05)$.

Abbreviation: miR-194, microRNA-194. 
A

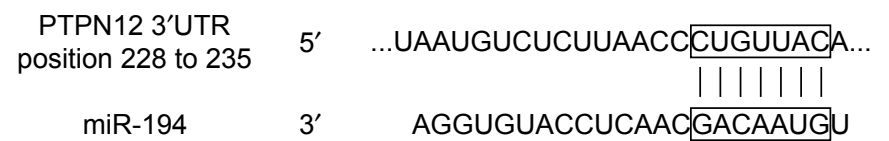

C

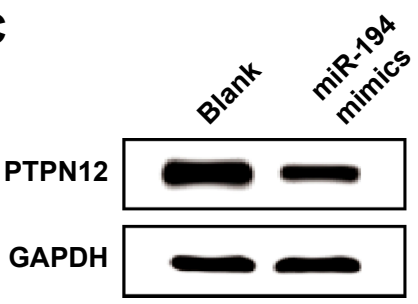

B

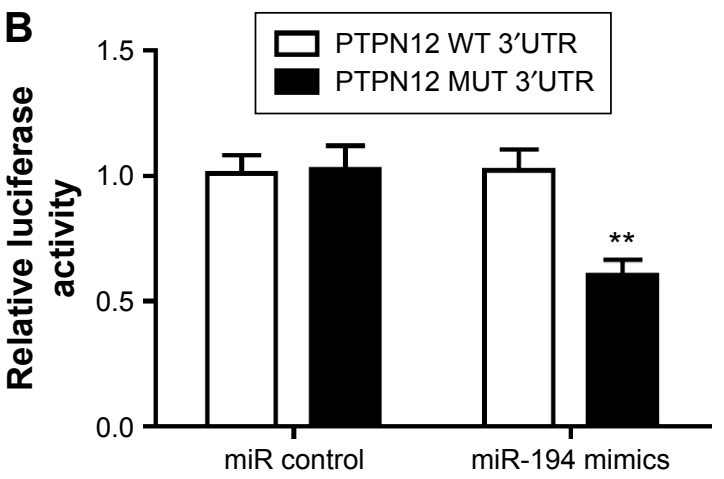

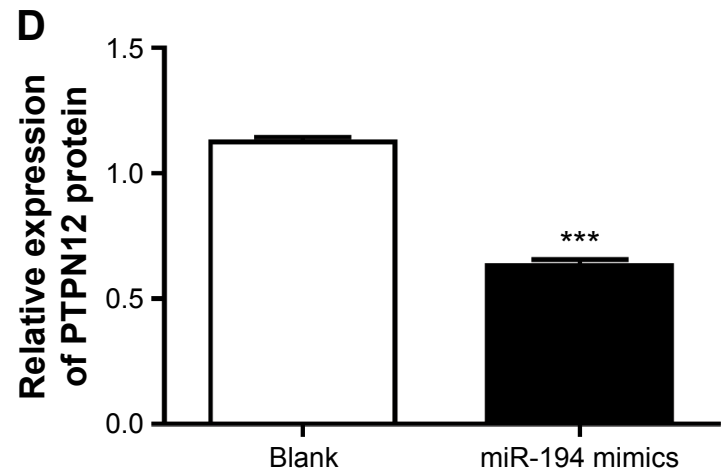

Figure 5 PTPNI2 is a direct target of miR-194 in ovarian cancer cells.

Notes: (A) Computational analysis identified that PTPNI 2 may be a potential target of miR-194, and the predicted binding sequences of PTPNI2 3'UTR and miR-194 were marked. (B) Relative luciferase activity was analyzed after PGL3-PTPNI2-WT and PGL3-PTPNI2-MUT plasmids were cotransfected with miR-194 mimics or control in SKOV3 cells. (C) Western blot assay of PTPNI 2 protein expression in SKOV3 cells transfected with miR-194 mimics and (D) graphical presentation. All experiments were performed in triplicate. ${ }^{*} * P<0.01$, $* * * P<0.001$.

Abbreviations: miR-194, microRNA-194; PTPNI2, protein tyrosine phosphatase nonreceptor type I2; UTR, untranslated region; WT, wild type; MUT, mutant.

that transfecting miR-194 mimics into ovarian cancer cells caused a significant decrease of PTPN12 on protein level (Figure 5C). Taken together, PTPN12 was directly regulated by miR-194. The real-time PCR assay showed that miR-194 mimics were statistically significantly at downregulating the protein expression of PTPN12 (Figure 5D).

\section{Discussion}

Ovarian cancer is still the most lethal gynecological malignancy worldwide. ${ }^{1}$ Its lethality may be due to the difficulties in early-stage detection and lack of effective treatments for patients with an advanced or recurrent status, ${ }^{28}$ and hence new ways for treatment and to induce metastasis of ovarian cancer to increase patients' survival rate are urgently needed. Recently, increasing evidences elucidated that miRNAs play a crucial role in biological and pathological process regulation, such as cell growth, tumor invasion, and metastasis. ${ }^{4,29}$ Many aberrant expressions of miRNAs have been reported in ovarian cancer. For example, members of the miR-200 family in ovarian cancer are downregulated, ${ }^{30,31}$ miR-199/214 cluster suppresses the tumorigenicity of ovarian cancer cells, ${ }^{32,33}$ meanwhile, let-7 family downregulation is involved in chemotherapy resistance in ovarian cancer cells and progression-free survival. ${ }^{31}$

miR-194 has been reported to play diverse roles in cancer cells. ${ }^{11,12,15,16}$ Aberrant expression of miRNAs is usually associated with tumor cell proliferation, invasion, and metastasis.
The varying function of miR-194 in different cancer cells was related to its different targets and the coregulation between them. ${ }^{29}$ miR-194 has been known to play an important role in regulating tumor growth and cell metastasis in colorectal cancer. However, the expression of miR-194 in ovarian cancer cells remains unclear. PTPN12 is a member of the PTP family, which is involved not only in cellular signaling but also in tumorigenesis. ${ }^{18,20}$ PTPN12 has been involved in regulation of cell adhesion and migration, ${ }^{34}$ and low expression of PTPN12 was often associated with tumor recurrence and poor prognosis. ${ }^{21-23}$ Our research found that miR-194 was upregulated and negatively associated with tumor suppressor protein PTPN12 in ovarian cancer tissues. These findings may explain the relation of miR-194 upregulation with poor survival of patients with ovarian cancer and provide a better understanding of the pathogenesis mechanisms and a potential therapeutic target of ovarian cancer.

In our research, we performed real-time PCR to detect the expression of miR-194 in tissues; results showed that compared with normal ovary tissues, miR-194 was overexpressed in ovarian cancer tissues, meanwhile, PTPN12 mRNA negatively regulated with miR-194. Furthermore, we verified the function of miR-194 on ovarian cancer cells. CCK-8 assays were performed to analyze cell growth in which we found that miR-194 mimics promoted cell growth in ovarian cancer cell lines. In addition, we 
analyzed cell migration and invasion by in vitro wound healing assay and transwell assay. Results showed that miR-194 enhanced cell migration and invasion. In contrast, miR-194 inhibitor affected the adverse functions of miR-194 mimics, namely, downregulated miR-194 decreased cell proliferation and reduced the capacity of cell migration and invasion. Importantly, we identified that in ovarian cancer cells, miR-194 directly binded to the 3'UTR of PTPN12; quantitative real-time PCR and Western blot analyses showed that miR-194 negatively regulated PTPN12 by reducing PTPN12 mRNA levels. However, how PTPN12 targeting by miR-194 leads to increased proliferation, migration, and invasion of ovarian cancer cells was not clear. Further studies on this regulation mechanism based on silencing PTPN12 are still needed.

\section{Conclusion}

We demonstrated that miR-194 was upregulated in ovarian cancer, which enhanced cell proliferation and metastatic capacity in ovarian cancer cells by targeting PTPN12. Thus, this new pathway may provide a new understanding on the mechanism of ovarian cancer. However, whether miR-194 targeting PTPN12 could be a new avenue for the treatment of ovarian cancer still needs to be further explored.

\section{Acknowledgment}

This study was supported by grants from the State Key Basic Research and Development Project of China (2012CB526700) and was also supported by the National Natural Science Foundation (No 81242092).

\section{Disclosure}

The authors report no conflicts of interest in this work.

\section{References}

1. Siegel RL, Miller KD, Jemal A. Cancer statistics, 2015. CA Cancer J Clin. 2015;65(1):5-29.

2. Berek JS, Crum C, Friedlander M. Cancer of the ovary, fallopian tube, and peritoneum. Int J Gynaecol Obstet. 2012;119:S118-S129.

3. Jayson GC, Kohn EC, Kitchener HC, Ledermann JA. Ovarian cancer. Lancet. 2014;384(9951):1376-1388.

4. Lee YS, Dutta A. MicroRNAs in cancer. Annu Rev Pathol. 2009;4: 199-227.

5. Esteller M. Non-coding RNAs in human disease. Nat Rev Genet. 2011; 12(12):861-874.

6. Zhong X, Coukos G, Zhang L. miRNAs in human cancer. Methods Mol Biol. 2012;822:295-306.

7. Berindan-Neagoe I, Monroig Pdel C, Pasculli B, Calin GA. MicroRNAome genome: a treasure for cancer diagnosis and therapy. CA Cancer J Clin. 2014;64(5):311-336.

8. He LT, Thomson JM, Hemann MT, et al. A microRNA polycistron as a potential human oncogene. Nature. 2005;435:828-833.
9. Xu Q, Liu LZ, Qian X, et al. MiR-145 directly targets p70S6K1 in cancer cells to inhibit tumor growth and angiogenesis. Nucleic Acids Res. 2012;40(2):761-774.

10. Taylor MA, Sossey-Alaoui K, Thompson CL, Danielpour D, Schiemann WP. TGF- $\beta$ upregulates miR-181a expression to promote breast cancer metastasis. J Clin Invest. 2013;123(1):150-163.

11. Dong P, Kaneuchi M, Watari H, et al. MicroRNA-194 inhibits epithelial to mesenchymal transition of endometrial cancer cells by targeting oncogene BMI-1. Mol Cancer. 2011;10:99.

12. Sundaram P, Hultine S, Smith LM, et al. p53-responsive miR-194 inhibits thrombospondin- 1 and promotes angiogenesis in colon cancers. Cancer Res. 2011;71:7490-7501.

13. Zhao HJ, Ren LL, Wang ZH, et al. MiR-194 deregulation contributes to colorectal carcinogenesis via targeting AKT2 pathway. Theranostics. 2014;4(12):1193-1208.

14. Wang B, Shen ZL, Gao ZD, et al. MiR-194, commonly repressed in colorectal cancer, suppresses tumor growth by regulating the MAP4K $4 / \mathrm{c}-$ Jun/MDM2 signaling pathway. Cell Cycle. 2015;14(7):1046-1058.

15. Li Z, Ying X, Chen H, et al. MicroRNA-194 inhibits the epithelialmesenchymal transition in gastric cancer cells by targeting FoxM1. Dig Dis Sci. 2014;59(9):2145-2152.

16. Zhang J, Zhao CY, Zhang SH, et al. Upregulation of miR-194 contributes to tumor growth and progression in pancreatic ductal adenocarcinoma. Oncol Rep. 2014;31:1157-1164.

17. Nakamura K, Sawada K, Kinose Y, Hashimoto K, Mabuchi S, Kimura T. Identification of microRNA which regulates paclitaxel resistance of ovarian cancer cells-a potential of miR-194 by attenuating paclitaxel resistance through the down-regulation of oncogene BMI-1. Cancer Res. 2014;74(19 suppl):4386.

18. Tonks NK. Protein tyrosine phosphatases: from genes, to function, to disease. Nat Rev Mol Cell Biol. 2006;7(11):833-846.

19. Espejo R, Rengifo-Cam W, Schaller MD, Evers BM, Sastry SK. PTPPEST controls motility, adherens junction assembly, and Rho GTPase activity in colon cancer cells. Am J Physiol Cell Physiol. 2010;299(2): C454-C463.

20. Streit S, Ruhe JE, Knyazev P, et al. PTP-PEST phosphatase variations in human cancer. Cancer Genet Cytogenet. 2006;170(1):48-53.

21. Luo RZ, Cai PQ, Li M, et al. Decreased expression of PTPN12 correlates with tumor recurrence and poor survival of patients with hepatocellular carcinoma. PLoS One. 2014;9(1):e85592.

22. Sun T, Aceto N, Meerbrey KL, et al. Activation of multiple protooncogenic tyrosine kinases in breast cancer via loss of the PTPN12 phosphatase. Cell. 2011;144(5):703-718.

23. Cao X, Li Y, Luo RZ, et al. Tyrosine-protein phosphatase nonreceptor type 12 is a novel prognostic biomarker for esophageal squamous cell carcinoma. Ann Thorac Surg. 2012;93(5):1674-1680.

24. Villa-Moruzzi E. Tyrosine phosphatases in the HER2-directed motility of ovarian cancer cells: involvement of PTPN12, ERK5 and FAK. Anal Cell Pathol (Amst). 2011;34(3):101-112.

25. Guo L, Zhao Y, Yang S, Zhang H, Chen F. An integrated analysis of miRNA, IncRNA, and mRNA expression profiles. Biomed Res Int. 2014;2014:345605.

26. Meng Z, Fu X, Chen X, et al. miR-194 is a marker of hepatic epithelial cells and suppresses metastasis of liver cancer cells in mice. Hepatology. 2010;52(6):2148-2157.

27. Livak KJ, Schmittgen TD. Analysis of relative gene expression data using real-time quantitative PCR and the 2(-Delta Delta C(T))method. Methods. 2001;25(4):402-408.

28. Kinose Y, Sawada K, Nakamura K, Kimura T. The role of microRNAs in ovarian cancer. Biomed Res Int. 2014;2014:249393.

29. Di Leva G, Garofalo M, Croce CM. MicroRNAs in cancer. Annu Rev Pathol. 2014;9:287-314.

30. Iorio MV, Visone R, Di Leva G, et al. MicroRNA signatures in human ovarian cancer. Cancer Res. 2007;67(18):8699-8707.

31. Nam EJ, Yoon H, Kim SW, et al. MicroRNA expression profiles in serous ovarian carcinoma. Clin Cancer Res. 2008;14(9):2690-2695. 
32. Yin $\mathrm{G}, \mathrm{Chen} \mathrm{R}$, Alvero AB, et al. TWISTing stemness, inflammation and proliferation of epithelial ovarian cancer cells through MIR199A2/214. Oncogene. 2010;29(24):3545-3553

33. Cheng W, Liu T, Wan X, Gao Y, Wang H. MicroRNA-199a targets CD44 to suppress the tumorigenicity and multidrug resistance of ovarian cancer-initiating cells. FEBS J. 2012;279(11):2047-2059.
34. Zheng Y, Yang W, Xia Y, et al. Ras-induced and extracellular signalregulated kinase 1 and 2 phosphorylation-dependent isomerization of protein tyrosine phosphatase (PTP)-PEST by PIN1 promotes FAK dephosphorylation by PTP-PEST. Mol Cell Biol. 2011;31(21): 4258-4269.

\section{Publish your work in this journal}

OncoTargets and Therapy is an international, peer-reviewed, open access journal focusing on the pathological basis of all cancers, potential targets for therapy and treatment protocols employed to improve the management of cancer patients. The journal also focuses on the impact of management programs and new therapeutic agents and protocols on

\section{Dovepress}

patient perspectives such as quality of life, adherence and satisfaction. The manuscript management system is completely online and includes a very quick and fair peer-review system, which is all easy to use. Visit http://www.dovepress.com/testimonials.php to read real quotes from published authors.

Submit your manuscript here: http://www.dovepress.com/oncotargets-and-therapy-journal 